\title{
ON ABSOLUTELY CONTINUOUS TRANSFORMATIONS
}

\author{
BY JAMES K. BROOKS AND PAUL V. REICHELDERFER
}

Communicated by Gian-Carlo Rota, June 16, 1969

1. Introduction. In this announcement, we examine absolutely continuous transformations $T$ mapping the measure space $(S, \Sigma, \mu)$ onto the measure space $\left(S^{\prime}, \Sigma^{\prime}, \mu^{\prime}\right)$. In order to obtain information about the change of measure induced by $T$, a weight function $W^{\prime}$ defined on $\boldsymbol{S}^{\prime} \times \mathfrak{D}$ is introduced, where $\mathfrak{D}$ is a certain subfamily of $\boldsymbol{\Sigma}$. $W^{\prime}\left(s^{\prime}, D\right)$ represents a weight assigned to the points in $D$ which $T$ maps into $s^{\prime} \in S^{\prime}$. We present structure theorems (Theorems 2 and 3) for weight functions which enable us to establish a transformation formula (Theorem 1) for integrals defined on the measure spaces. Theorem 1 includes all the existing transformation formulas for transformations which are absolutely continuous with respect to a real valued weight function. Moreover, the integrability condition necessary to ensure the existence of the formula is minimal, as we shall indicate in $\S 3$.

Rado and Reichelderfer [11] considered the case when the measure spaces are Euclidean $n$-space (both having the same dimension), with Lebesgue measurable sets and $n$-dimensional Lebesgue measure; $T$ is a bounded continuous transformation defined on the bounded domain $S$. In particular, the weight function $\mu_{e}\left(s^{\prime}, T, D\right)$ generated by the topological index defined on indicator domains is used to define an essentially absolutely continuous transformation. Also the Banach indicatrix or crude multiplicity function $N\left(s^{\prime}, T, D\right)$ and the weight function $k\left(s^{\prime}, T, D\right)$ which counts the number of essential maximal model continua for $\left(s^{\prime}, T, D\right)$ are treated in detail in [11]. In this classical setting, Craft [10] removed some conditions on the weight functions. Reichelderfer [13] developed a transformation theory for general measure spaces under certain standard hypotheses. Necessary and sufficient conditions were given in order that a transformation be absolutely continuous. In [14] it was shown that a large class of topological spaces satisfies these hypotheses; consequently, in this general topological setting the concepts of absolute continuity and generalized Jacobians can be effectively defined. Brooks [1], [3] developed the theory for integrals in Banach spaces and introduced a larger class of weight functions [2]; as a special case, signed weight functions may now be used when the spaces are oriented. Lebesgue decomposition theorems and measurability theorems for positive weight functions were considered by Chaney [6], [7], [8]. 
The theorems in $\S 3$ will be used at a later date to develop a chain rule for the product of absolutely continuous transformations. This problem was first solved for Euclidean $n$-space in [12] and was treated in a more general setting in [9].

2. The setting. In this section we establish notation and state some definitions. Throughout this paper we assume that the standard hypotheses for transformation theory $\mathrm{H} 1-\mathrm{H} 8$ are satisfied (see [13] or [1]). For the reader's convenience some of the families of sets occurring in these hypotheses are listed.

$(S, \Sigma, \mu)$ and $\left(S^{\prime}, \Sigma^{\prime}, \mu^{\prime}\right)$ are $\sigma$-finite complete measure spaces. $T$ is a function (transformation) mapping $S$ onto $S^{\prime}$. D is a subfamily of $\Sigma$ containing $\varnothing$ and $S$. $D$ will be a generic notation for a set in $\mathfrak{D}$. $T \mathfrak{D} \subseteq \Sigma^{\prime}$ and the intersection of two sets in $\mathfrak{D}$ can be written as a countable union of disjoint sets from $\mathfrak{D}$. For every $E \in \Sigma$ and $\epsilon>0$ there exists a disjoint sequence $\left\{D_{i}\right\}$ such that $E \subseteq \cup D_{i}$ and $\mu\left(\cup D_{i}-E\right)<\epsilon$. A set $E \subseteq S$ is $\mu \mu^{\prime}$-null if $E=A_{1} \cup A_{2}$, where $\mu\left(A_{1}\right)$ $=\mu^{\prime}\left(T A_{2}\right)=0 . \Delta(D)\left(\Delta^{*}(D)\right)$ denotes the family of all finite (countable) collections of pairwise disjoint sets in $\mathscr{D}$ contained in $D$. $R$ denotes the real numbers.

A signed weight function for $T$ is a real valued function $W^{\prime}$ defined on $\boldsymbol{S}^{\prime} \times \mathfrak{D}$ such that:

(i) $W^{\prime}(\cdot, D)=0$ a.e. $\mu^{\prime}$ on $S^{\prime}-T D$;

(ii) If $D_{i} \uparrow D$, then $\lim W^{\prime}\left(\cdot, D_{i}\right)=W^{\prime}(\cdot, D)$ a.e. $\mu^{\prime}$;

(iii) If $\left\{D_{i}\right\} \in \Delta^{*}(D)$ and $D-\cup D_{i}$ is $\mu \mu^{\prime}$-null, then $W^{\prime}(\cdot, D)$ $=\sum W^{\prime}\left(\cdot, D_{i}\right)$ a.e. $\mu^{\prime}$;

(iv) $W^{\prime}(\cdot, D)$ is measurable for each $D$. $W^{\prime}$ will always denote a signed weight function.

A positive weight function $G^{\prime}$ is a nonnegative function satisfying the above conditions except (iii) is replaced by the requirement that $\sum G^{\prime}\left(\cdot, D_{i}\right) \leqq G^{\prime}(\cdot, D)$ a.e. $\mu^{\prime}$, whenever $\left\{D_{i}\right\} \in \Delta^{*}(D)$. Write $G_{1}^{\prime} \prec G_{2}^{\prime}$ if $G_{1}^{\prime}(\cdot, D) \leqq G_{2}^{\prime}(\cdot, D)$ a.e. $\mu^{\prime}$ for every $D$.

$T$ is of bounded variation with respect to $W^{\prime}\left(B V W^{\prime}\right)$ if there exists a nonnegative function $K^{\prime} \in \mathscr{L}_{1}\left(\mu^{\prime}\right)$ such that for $\left\{D_{i}\right\} \in \Delta^{*}(S)$, $\sum\left|W^{\prime}\left(\cdot, D_{i}\right)\right| \leqq K^{\prime}$ a.e. $\mu^{\prime} . T$ is absolutely continuous with respect to $W^{\prime}\left(A C W^{\prime}\right)$ if $T$ is $B V W^{\prime}$ and there exists a function $f \in \mathscr{L}_{1}(\mu)$ such that $\int_{D} f d \mu=\int_{T D} W^{\prime}(\cdot, D) d \mu^{\prime}$, for every $D . f$ is called a generalized Jacobian for $T$ relative to $W^{\prime}$ (it follows that $f$ is unique in $\mathscr{L}_{1}(\mu)$ ). A function $g$ satisfies condition $(N)_{T}$ if $g=0$ a.e. $\mu$ on $T^{-1} E^{\prime}$ whenever $\mu^{\prime}\left(E^{\prime}\right)=0$. Let $W(D)=\int_{T D} W^{\prime}(\cdot, D) d \mu^{\prime}, D \in \mathfrak{D}$. Define

$$
V(D, W)=\sup _{\Delta(D)} \sum\left|W\left(D_{i}\right)\right| ; V^{\prime}(\cdot, D)=\sup _{\Delta(D)} \sum\left|W^{\prime}\left(\cdot, D_{i}\right)\right| .
$$


The above definitions include the existing definitions of bounded variation and absolute continuity in the literature. We assume in the sequel that $T$ is $A C W^{\prime}$ with generalized Jacobian $f$.

3. The results. Proofs for the theorems in this section will appear elsewhere. The main result is the following

Theorem 1 (TRANSFormation Formula). Let $H^{\prime}: S^{\prime} \rightarrow R$ be measurable. Then $H^{\prime} \circ T f$ is measurable. If $H^{\prime} \circ T f$ is $\mu$-integrable on a fixed set $D$, then $H^{\prime} W^{\prime}(\cdot, D)$ is $\mu^{\prime}$-integrable and

$$
\int_{D} H^{\prime} \circ T f d \mu=\int_{T D} H^{\prime} W^{\prime}(\cdot, D) d \mu^{\prime} .
$$

As mentioned above, this theorem extends the results of Rado and Reichelderfer in Euclidean $n$-space [11, p. 262]. We mention that the integrability of $H^{\prime} W^{\prime}(\cdot, D)$ does not imply the integrability of $H^{\prime} \circ T f$ on $D[4]$; however, the integrability of $H^{\prime} V^{\prime}(\cdot, D)$ implies the integrability of $H^{\prime} \circ T f$ on $D$ [2]. The following results which are used to establish Theorem 1 are interesting in their own right.

THEOREM 2. $f$ satisfies condition $(N)_{T}$ and $V(D, W)=\int_{D}|f| d \mu$.

The proof of the first assertion involves a technique similar to the one found in the proof of Lemma 3.1 in [2]. The proof of the second part is a long technical argument using $\gamma$-type partitions of elements of $\mathfrak{D}[13]$.

The next theorem yields a decomposition for weight functions which complements the pointwise decomposition theorems presented in [5].

TheOREM 3 (JoRdAN DECOMPOSITION). There exist positive weight functions $Q^{\prime}, Q_{ \pm}^{\prime}$ for $T$ such that

1. $Q^{\prime}=Q_{+}^{\prime}+Q_{-}^{\prime} ; W^{\prime}=Q_{+}^{\prime}-Q_{-}^{\prime}$.

2. $T$ is $A C Q^{\prime}, A C Q_{ \pm}^{\prime} ;|f|\left(f^{ \pm}\right)$is generalized a Jacobian for $T$ relative to $Q^{\prime}\left(Q_{ \pm}^{\prime}\right)$.

3. If $U^{\prime}, U_{ \pm}^{\prime}$ are nonnegative real valued functions defined on $\boldsymbol{S}^{\prime} \times D$ such that $W^{\prime}=U_{+}^{\prime}-U_{-}^{\prime}$ and $U^{\prime}=U_{+}^{\prime}+U_{-}^{\prime}$ is a positive weight function for which $T$ is $A C U^{\prime}$, then $U_{ \pm}^{\prime}$ are positive weight functions, $T$ is $A C U^{\prime}$, and $U_{ \pm}^{\prime} \succ Q_{ \pm}^{\prime}$.

In the proof, the function $\Psi_{D}\left(E^{\prime}\right)=\int_{\left(T^{-1} E^{\prime}\right) \cap D}|f| d \mu, E^{\prime} \in \Sigma^{\prime}$ is used (cf. [8]). $\Psi_{D}$ is well defined on $\Sigma^{\prime}$ and $\Psi_{D} \ll \mu^{\prime}$ by Theorem 2. Hence, we can define $Q^{\prime}(\cdot, D)=\left(d / d \mu^{\prime}\right) \Psi_{D} . Q_{ \pm}^{\prime}$ are defined by 1 . By using 
convergence theorems, one can show that $Q^{\prime}, Q_{ \pm}^{\prime}$ satisfy the conclusions of the above theorem.

\section{BIBLIOGRAPHY}

1. J. K. Brooks, Transforming bilinear vector integrals, Studia Math. 33 (1969), 159-165.

2. - On absolute continuity in transformation theory, Monatsh. Math. 73 (1969) 1-6.

3. - Transformation theory for vector measure spaces, An. Acad. Brasil Ci. 41 (1969), 15-18.

4. - On generalized Jacobians, Funkcial. Ekvac. (1) 12 (1969), 1-6.

5. J. K. Brooks and P. V. Reichelderfer, A Jordan decomposition for weight functions, Rend. Circ. Mat Palermo (to appear).

6. R. W. Chaney, Measurability theorems in the transformation theory for measure space, Rend. Circ. Mat. Palermo 14 (1965), 309-323.

7. - Decomposition theorems for weight functions in the transformation theory for measure space, Rend. Circ. Mat. Palermo 15 (1966), 98-128.

8. - On the transformation of integrals in measure space, Pacific J. Math. (2) 19 (1966), 229-242.

9. - A chain rule for the transformation of integrals in measure space, Pacific J. Math. 25 (1968), 33-57.

10. G. A. Craft, A transformation theory for weight functions, Rend. Circ. Mat. Palermo 10 (1961), 212-228.

11. T. Rado and P. V. Reichelderfer, Continuous transformations in analysis, Springer-Verlag, Berlin, 1955.

12. P. V. Reichelderfer, On the product of absolutely continuous transformations in Euclidean n-space, Rend. Circ. Mat. Palermo 5 (1956), 5-42.

13. - A transformation theory for measure space, Rend. Circ. Mat. Palermo 10 (1961), 283-313.

14. —, A transformation theory for topological space, Math. Japon. 7 (1962), 181-194.

UNIVERSity of Florida, GaINEsville, Florida 32601 aNd

Ohio University, Athens, Ohio 45701 